\title{
Chronic disease management in
} primary health care: The role of rehabilitation professions

\author{
Danielle Naumann
}

\section{Queen's University}

The manner in which Canadians access primary health care services is in the midst of major reform due to changing demographic trends that place increased demands on the primary care system. Canada's federal, provincial, and territorial governments have committed to ensuring the provision of competent and accessible health care services in a cost-efficient and patient-centred manner that addresses these trends: an aging population, decreased interest in the practice of family medicine, a shift from hospital to community care and increased prevalence of chronic diseases. ${ }^{1,2}$ Chronic diseases are non-communicable and incurable long-term illnesses that become progressively severe over time. Patients with multiple chronic diseases consume disproportionately more health care resources: they require intensive management and coordination between numerous service providers, frequent contact with their physician, and a significant degree of ongoing coaching and support. ${ }^{3}$ Consequently, these patients face fragmented and incomplete care, which results in the development of secondary disabilities and comorbidities. More patients with complex conditions are seeking primary care to address their ongoing health care needs. These patients represent $5-6 \%$ of primary care caseloads, and consume a third of practice resources, ${ }^{3}$ resulting in a need for rehabilitation professions to offset this burden. Skilled in both prevention and intervention as they relate to patientcentred chronic disease management, rehabilitation professions are a valuable asset to primary care. This article introduces the utility of rehabilitation in primary care in order to facilitate effective and efficient health care services to patients at risk for or diagnosed with a chronic disease.

\section{What are rehabilitation professions?}

Rehabilitation professions are founded on common goals and philosophies including patient-centered holistic practice, health promotion, functional improvement and improved quality of life. ${ }^{5,6}$ In the primary care setting, they provide preventative health services by screening for disease and intermediate risk conditions, and health education programmes that provide education on the impact of unhealthy behaviour. ${ }^{7}$ They offer community development services by facilitating collective action in order to identify and meet widespread health needs. They are also engaged in public health policy and environmental health policy, advocating for changes facilitating positive health behaviour and enforcing improvements to environmental amenities.

The practice tools of rehabilitation professions, such as physical therapists and occupational therapists, can enhance traditional biomedical treatments, and engage the patient in actively addressing factors in their environment and health behaviours that contribute to the experience of disability. ${ }^{7-9}$ Physiotherapists address the physical risk factors and intermediate conditions associated with chronic disease by enabling patients to engage in physical activity and mobility. ${ }^{5}$ Occupational therapists use activities that are familiar to patients to promote personal responsibility for health, and are leaders in facilitating community development; conducting assessment and screening related to occupational performance; offering counselling, coaching and consultation; and bridging health systems to facilitate integrated health care. ${ }^{6}$

\section{How can rehabilitation professions improve primary care?}

The traditional health care system focuses on reactive responses to acute illness and cannot provide patients with chronic diseases with the standard of care expected under the Canada Health Act. $^{3}$ In order to manage the disease process in a manner associated with optimal outcomes and the greatest quality of life, patients with a chronic disease need to receive rehabilitation interventions early in the disease process; however, many Canadians living with chronic conditions continue to experience excessive wait 
c times for rehabilitation services due to limited availability

in primary care settings. . $^{2-4}$

Government-supported movement from traditional feefor-service models to collaborative multidisciplinary models has helped to improve patient access to primary health care. It has also provided physicians with incentives and funding resources for which to offer competent care to complex patients, lifting the burden to the health care system. ${ }^{8,9}$ By providing incentives to build a team approach to providing comprehensive primary care, this support has been instrumental to the inclusion of rehabilitation professions in multidisciplinary teams. ${ }^{2-4}$ Researchers and clinicians are in agreement that the inclusion of rehabilitation in primary health care delivery (e.g. Family Health Teams and Community Health Centres) is associated with a range of benefits: ${ }^{8}$ decreased waiting time, reduced specialist referrals, lower costs for services, greater continuity of care, improved patient outcomes in measures of quality of life, exercise tolerance, treatment compliance, self-management and/or improved health status. ${ }^{1-4,7-10}$ Rehabilitation is integral to the provision of primary health care delivery that offers comprehensive, accessible, and patient-centred interventions and support for chronic disease. $^{10}$

Rehabilitation professionals are experts in both the prevention and management of chronic disease, and are ideally positioned to enhance the capacity and infrastructure of the primary care system through the delivery of cost-efficient and effective chronic disease management. Movement toward widespread inclusion of occupational therapy and physiotherapy in primary care across Canada will address increasing prevalence of chronic disease and provide these patients with equitable access to the standard of care outlined in the Canada Health Act.

\section{References}

1. Mable A, Marriott J. Sharing the learning-the health transition fund synthesis series: primary health care. Ottawa: Health Canada; 2002.

2. Cott C, Devitt R, Wong R, Soever L, MacKay, C. A client-centred health service model of primary health care and rehabilitation for arthritis. Toronto: Arthritis Community Resource Evaluation Unit; 2005.

3. Wallace $P$, Seidman J. Improving population health and chronic disease management. In Dorland J, McColl MA, editors. Emerging approaches to chronic disease management in primary health care. Montreal and Kingston: McGill-Queen's University Press; 2007. p. 15-20.

4. Passalent LA, Landry MD, Cott CA. Wait times for publicly funded outpatient and community physiotherapy and occupational therapy services: implications for the increasing number of persons with chronic conditions in Ontario, Canada. Physiother Can. 2009; 61(1): 5-14.

5. Canadian Physiotherapy Association (CPA). About physiotherapy [Internet]. Ottawa: Canadian Physiotherapy Association; 2012 [last update unknown; cited 2014 Mar 20]. Available from: http://www.physiotherapy.ca/AboutPhysiotherapy

6. Leclair L, Restall G, Edwards J, Cooper JSM, Soltys P, Sapacz R. Occupational therapists and primary health care: position paper [Internet]. Winnipeg: Manitoba Society of Occupational Therapists; 2005 [last update unknown; cited 2014 Mar 20]. Available from: http://www.msot.mb.ca/uploads/ PositionPaper_PrimaryHealthCare.pdf

7. Gottwald M. Health promotion models. In Davis S, editor. Rehabilitation: the use of theories and models in practice. Toronto: Elsevier; 2005. p. 131-146.

8. Donnelly C, Brenchley C, Crawford C, Letts L. The integration of occupational therapy into primary care: a multiple case study design. BMC Fam. Pract. 2013; 14(1): 60 .

9. Kralj B, Kantarevic J. Primary care in Ontario: reforms, investments and achievements. Ont Med Rev. 2012; 79(2): 18-24.

10. Eldar R. Integrated institution - community rehabilitation in developed countries: a proposal. Disabil. Rehabil. 2000; 22(6): 266-274.

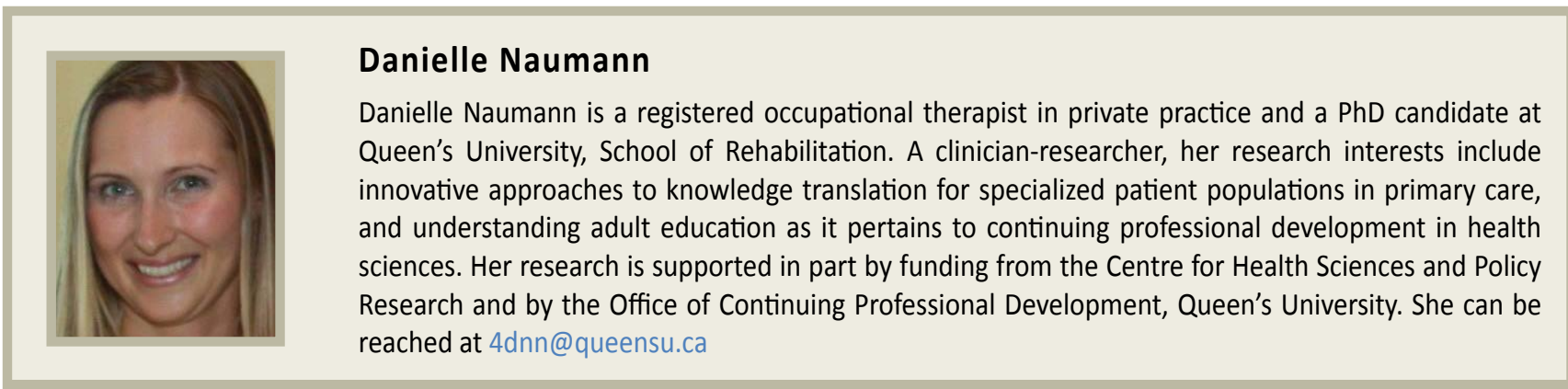

